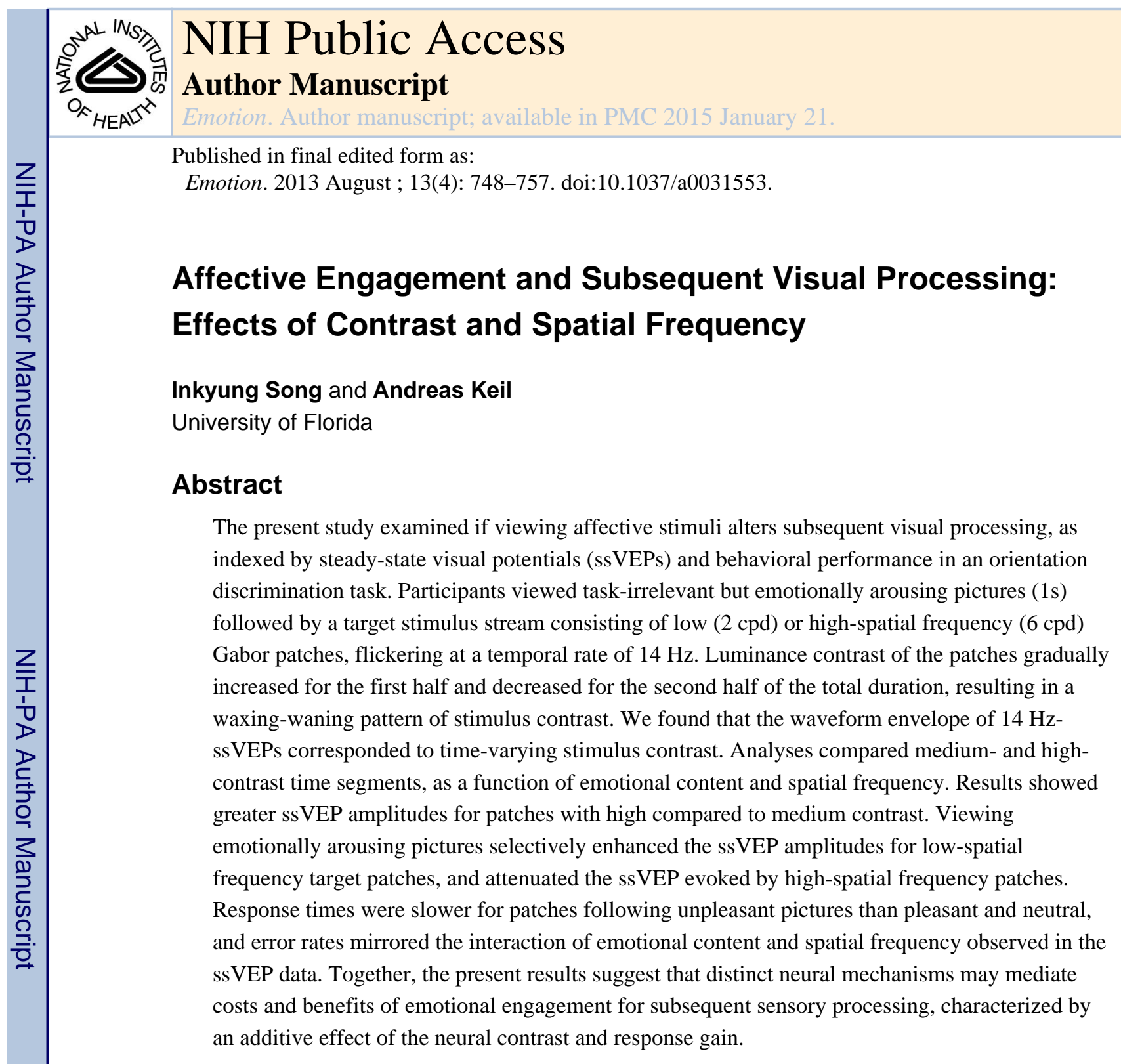

\title{
Keywords
}

steady-state; emotion; subsequent visual processing; contrast; spatial frequency

\begin{abstract}
Adaptive behavior requires the selection and evaluation of relevant visual information in space and time. Because the capacity of sensory systems is limited, mechanisms that prioritize relevant information are essential for eliciting adaptive responses (Vuilleumier, 2005). Over many decades, this work has converged to show that attention not only selects relevant stimuli from the array presented to the sensory organ, but also actively shapes early visual processing of attended information to meet current behavioral requirements (D. Lee, Koch, \& Braun, 1997; Reynolds, Pasternak, \& Desimone, 2000).
\end{abstract}

Correspondence concerning this article should be addressed to Inkyung Song, Center for the Study of Emotion \& Attention, University of Florida, PO Box 112766, Gainesville, FL 32611, Phone: 352-392-2439, Fax: 352-392-6047, Contact: isong@ufl.edu. 
In the visual system, neurophysiological evidence from animal studies has shown stronger neuronal responses, when viewing task-relevant, compared to irrelevant, stimuli (Desimone \& Ungerleider, 1989). Notions that attention affects sensory processes have been consistently supported by human psychophysical (Carrasco, Ling, \& Read, 2004; Huang \& Dobkins, 2005; D. Lee, et al., 1997) and functional imaging studies (Corbetta et al., 1990; Kastner et al., 1999) on a range of perceptual dimensions, such as contrast sensitivity and spatial resolution. Here, we ask how the perception of an emotionally engaging stimulus affects subsequent perceptual sensitivity, as measured by means of electrophysiology.

\section{Attention and Emotion}

Attention can be voluntarily directed to target visual stimuli for specific task performance or automatically driven by virtue of motivational properties of a stimulus (Ferrari, Codispoti, Cardinale, \& Bradley, 2008). In particular, affectively arousing properties of words, natural scenes, and facial expressions have been suggested to automatically attract attentional resources to facilitate sensory processing (Keil et al., 2005). This is consistent with an evolutionary perspective on survival or reproduction (Dolan, 2002; Phelps \& LeDoux, 2005), such that emotional stimuli may be granted priority for a preferential perceptual analysis (B. Bradley et al., 1999; Stormark, Nordby, \& Hugdahl, 1995) in order to promote appropriate behavioral responses to changes in the environment. Empirically, behavioral studies have reported more rapid and accurate identification of arousing stimuli than neutral ones using attention blink (Anderson \& Phelps, 2001; Keil \& Ihssen, 2004), visual search (Ohman, Flykt, \& Esteves, 2001), and choice-reaction tasks (Zeelenberg, Wagenmakers, \& Rotteveel, 2006). Recent brain imaging and psychophysiological studies have also indicated that emotional significance of stimuli facilitated the early stage of visual processing (M. Bradley et al., 2003; Keil et al., 2008).

In terms of neurophysiological mechanisms, afferent signals from anterior cortical and subcortical structures have been proposed to provide modulatory input into visual cortex (i.e., the re-entrant hypothesis). In macaque monkeys, for example, neurons in the magnocellular division of the basal nucleus of the amygdala project to the visual system and innervate all occipital and temporal levels, whereas those in the intermediate and parvocellular division project to more rostral and medial portions of the visual cortex, suggesting multiple sources capable of altering sensory processing (Amaral, Behniea, \& Kelly, 2003). In line with findings from animal models, human amygdaloid complex and parietofrontal cortex have been implicated in facilitated visual perception of affectively arousing stimuli, as indexed by electrocortical (Keil et al., 2007) and hemodynamic responses (Pessoa, McKenna, Gutierrez, \& Ungerleider, 2002; Sabatinelli et al., 2004).

\section{Emotion and Concurrent/Subsequent visual processing}

The aforementioned attentional and perceptual advantages for emotional stimuli raise the question of their costs and benefits when an observer views multiple stimuli, potentially representing competing task demands. For instance, emotional pictures have been found to strongly interfere with a concurrently presented task stimulus that overlaps with an emotional distractor (Muller, Andersen, \& Keil, 2008). Behavioral and psychophysiological 
findings have shown that the emotional significance of prior visual stimuli (e.g., words, pictures, and faces), regardless of their task-relevance, may alter the amount of sensitivity to subsequent target items, respectively (Bocanegra \& Zeelenberg, 2009b, 2011). Interestingly, both emotional interference and facilitation on subsequent task performance has been reported in these paradigms:

Phelps et al. (2006) presented fearful or neutral face cues prior to peripheral Gabor patches varying in contrast and orientation. The authors reported that a valid cue (i.e., a fearful/ neutral face at the spatial location of the upcoming Gabor stimuli) enhanced the orientation discrimination of the target Gabor patch. Importantly, the beneficial effect of attention on contrast sensitivity (i.e., the difference in behavioral performance between valid and invalid spatial cues) was greater when the target followed fearful face cues. Thus, emotional stimuli, which rapidly and automatically attract additional attentional resources, may exert a general beneficial carryover effect on subsequent perceptual processing of elementary visual features, such as luminance contrast (Phelps, et al., 2006).

By contrast, other studies have demonstrated that the processing of visual stimuli in spatial (Muller, et al., 2008) or temporal proximity (Most et al., 2007) to an emotional stimulus is impaired. This has often been related to limited attentional resources, being preferentially allocated to an emotionally arousing stimulus (Bocanegra \& Zeelenberg, 2009a). Using a modified attentional-blink paradigm (Chun \& Potter, 1995; Raymond, Shapiro, \& Arnell, 1992) within a rapid stream, studies have also addressed the perceptual bias toward emotionally arousing pleasant pictures (Most, et al., 2007) and aversively conditioned neutral pictures (Smith, Most, Newsome, \& Zald, 2006) at the expense of a neutral target stimulus.

Recently, researchers have sought to identify parameters that mediate costs versus benefits of emotional engagement not only on attentive processing, but also on perceptual processing of subsequent visual information. For example, emotional words impaired target identification at short latencies (50 and $500 \mathrm{~ms}$ ), but facilitated identification at long interstimulus intervals of $1000 \mathrm{~ms}$ (Bocanegra \& Zeelenberg, 2009a). A recent behavioral study reported that task-irrelevant emotional cues (i.e., fearful faces) selectively enhanced correct identification of target Gabor patches with coarse low spatial-frequency (LSF), but impaired the processing of those with fine-grained high spatial-frequency (HSF(Bocanegra \& Zeelenberg, 2009b). Bocanegra and Zeelenberg (2011) further examined the trade-off effect of emotion on spatial and temporal resolution, taking their results to suggest inhibitory interactions between magnocellular and parvocellular type channels in the visual system. According to the authors, slower and sustained responses of parvo cells are selective for fine-grained HSF stimuli at high luminance contrast, whereas fast and transient responses of magno cells are selective for coarse LSF stimuli at low contrast. Especially in regard to the reciprocal connectivity between the magnocellular division of amygdala and visual cortices (Amaral, et al., 2003), an early facilitation of magnocellular processing induced by emotional stimuli may accompany an inhibition of parvocellular processing (Bocanegra \& Zeelenberg, 2011). 


\section{How emotion may affect perception: Gain models of attention}

At least two different mechanisms have been identified in the extant literature as to how activity in visual cortical networks may be altered to modulate perceptual sensitivity: the contrast gain and response gain models of attention. According to the contrast gain hypothesis, attention increases neuronal responses when stimulus contrast is below the point of response saturation of the dynamic range of the neural contrast-response function (CRF, where a response measure is shown on the ordinate and stimulus contrast is shown on the abscissa; e.g., (Kim et al., 2007). Thus, a specific modulation of the CRF in the medium contrast range, where responses are neither noise nor at saturation, is typically regarded as evidence for contrast gain. Conversely, the response gain hypothesis postulates that attention multiplicatively boosts the activity of sensory neurons, leading to increases of the CRF across the entire dynamic range and changing the maximum response (see Figure 1, Panel A). According to Ling and Carrasco (2005), contrast gain predicts an increase in sensitivity (i.e., a threshold shift in the CRF, see Figure 1, Panel B), whereas response gain predicts an overall increase in firing rate (i.e., changes in slope and asymptote).

Supporting the contrast gain hypothesis, several single-cell recording (e.g., Martinez-Trujillo \& Treue, 2002; Reynolds, et al., 2000) and human psychophysical studies (e.g., Carrasco, et al., 2004) have reported a stronger attentional effect for stimuli with medium luminance contrast, compared to those with low or high contrast. Other psychophysical studies have demonstrated an increase in the asymptote of the CRF without response saturation, supporting a multiplicative enhancement of neuronal responses (i.e., response gain) that leads to the greatest attention effect for stimuli with high luminance contrast (Morrone, Denti, \& Spinelli, 2004). Many empirical studies have found evidence for both contrast and response gain, suggesting that the specific mechanism supported in a given study may depend on factors such as the type of attentional selection (spatial, feature, or object based), task duration, or visual stimulus properties. These reports have converged to demonstrate that contrast and response gain may be observed in different experimental designs, or even in different conditions within one experimental design (see Carrasco, 2011 for review). This line of work has led researchers to propose a mixed model of contrast and response gain that modulate the attention effect on perceptual sensitivity of contrast-varying stimuli (see Figure 1, Panel C). For instance, an additive shift of the CRF during spatial attention has often been observed, and can be taken to reflect both contrast and response gain, possibly including increased baseline activity (Buracas \& Boynton, 2007; Li et al., 2008). Moreover, other studies have demonstrated that contrast gain affects the early stage of processing, followed by response gain at the later stage in transient/exogenous attention (Ling \& Carrasco, 2006) and sustained attention tasks (Huang \& Dobkins, 2005).

\section{The Present Study}

In the present study, we examined the neurophysiological mechanisms of low-level visual processing subsequent to emotional engagement using affective images. As a continuous measure of lower-tier visual cortical activity in response to a target stimulus stream, we employed the steady-state visual evoked potential (ssVEP; Regan, 1989). The ssVEP is an oscillatory response of the visual cortex elicited e.g., by luminance-modulated stimuli with a 
fixed frequency, resulting in high signal-to-noise ratio information potentially modulated by visual selective attention (Muller et al., 1998). Given these methodological advantages, the ssVEP is expected to be sensitive to both costs (visual resource allocation is attenuated) and benefits (visual resource allocation is enhanced) of emotional engagement on subsequent visual processing. Considering that perceptual processing is sensitive to the physical properties of an attended stimulus (brightness, contrast, color, etc.), we addressed three groups of hypotheses regarding the impact of emotional engagement on subsequent electrocortical sensitivity of early visual areas during a perceptual task using Gabor patch varying in contrast and spatial frequency.

First, emotional engagement influences the subsequent visual processing of neutral targets. If emotion enhances subsequent visual processing, then higher ssVEP amplitudes for a target following a pleasant and unpleasant picture than a neutral one should be observed. Alternatively, if the perceptual benefit is restricted to an emotional stimulus itself and impairs the subsequent visual processing of task-relevant stimuli, lower ssVEP amplitudes should be observed for a target following emotionally arousing, compared to neutral, pictures.

Second, it was expected that the emotional facilitation or attenuation of early visual cortical activity systematically varies with the time-varying stimulus contrast: If the amplitude difference between emotional and neutral cue conditions is greater for medium than high contrast gratings, then the contrast gain model would be supported. Conversely, a greater amplitude difference of the ssVEP when viewing high-contrast gratings, compared to medium-contrast stimuli, would support the response gain model. If a similar emotional effect on the ssVEP amplitude when viewing medium and high contrast gratings is observed, the mixed model (i.e., an additive pattern of contrast and response gain, see Figure 1, Panel C) would be supported.

Finally, the literature reviewed above suggests differential connectivity patterns of the magnocellular and parvocellular visual pathways with deep structures sensitive to emotional content (e.g., the amygdala). The extent to which these deep structures modulate perception following emotional engagement may be selective for differential stimulus properties of a target stimulus that are predominantly conveyed through each visual pathway. Specifically, the difference in ssVEP amplitudes between emotional and neutral cue conditions may depend on the pathway that is primarily engaged by luminance contrast and spatial frequency of grating stimuli. Given previous findings with stimuli designed to specifically engage magnocellular and parvocellular pathways, we expect that visual cortical activity subsequent to emotional engagement varies as a function of magnocellular (conveyed through low spatial frequency) versus parvocellular biases, conveyed through high spatial frequency of the contrast-varying Gabor patch.

\section{Methods}

\section{Participants}

49 undergraduate students ( $65 \%$ female; age $\mathrm{M}=19.06$ years, $\mathrm{SD}=1.05$ ) of the University of Florida participated for psychology course credit. All Participants were screened for a 
personal and family history of photic epilepsy. Experimental procedures were approved by the institutional review board of the University of Florida, and participants gave written informed consent.

\section{Stimuli}

All visual stimuli were generated and presented using MATLAB (R2007b; Mathworks, Inc., Natick, MA, USA) and the Psychophysics Toolbox (Brainard, 1997; Pelli, 1997). They were displayed centrally with a white fixation cross on a uniform gray background on a 19-inch computer monitor with a vertical refresh rate of $70 \mathrm{~Hz}$. The target stimuli consisted of a continuous stream of slightly tilted ( $2^{\circ}$ clockwise or counterclockwise) Gabor patches, presented in a 14-Hz flickering mode (i.e. alternating with the gray background 14 times per second). Luminance contrast of the patches gradually and linearly increased and decreased over time from a Michelson contrast of 0 to .9 , and back to 0 , resulting in a waxing-waning pattern of stimulus contrast on a trial basis. The stream of Gabor patches had either low (2 cpd) or high (6 cpd) spatial frequency. To manipulate the emotional content of a taskirrelevant cue preceding the flickering stream of the Gabor patches, sixty pictures (20 pleasant, neutral, unpleasant, respectively) were selected from the IAPS (Lang, Bradley, \& Cuthbert, 2005) based on normative valence and arousal ratings from the Self-Assessment Manikin (SAM; M. Bradley \& Lang, 1994) 9-point scale. Mean (Standard Deviation) of valence and arousal ratings for neutral pictures were 5.24 (1.28) and 3.28 (1.99), for pleasant 7.30 (1.53) and 5.36 (2.18), and for unpleasant 1.96 (1.41) and 6.85 (2.21), respectively. Converted to grayscales, all pictures were presented twice throughout the experiment, resulting in total 120 trials.

\section{Design and Procedure}

Participants viewed the display at a distance of $57 \mathrm{~cm}$ and were instructed to fixate at the center of the screen, marked by the central fixation cross, throughout the experiment. The experimental design involved an orientation discrimination task during the presentation of each flickering stream of tilted Gabor patches. The sequence of stimulus presentation is illustrated schematically in Figure 2. After the display of a central fixation cross (500 ms), a picture cue with different emotional contents (i.e., pleasant, neutral, and unpleasant) was presented for $1000 \mathrm{~ms}$, followed by a gray screen for $600 \mathrm{~ms}$. Finally, the target stream of flickering Gabor patches appeared at the center of the screen for a duration of $5600 \mathrm{~ms}$. One of the two spatial frequencies of Gabor patches, either low ( $2 \mathrm{cpd}$ ) or high ( $6 \mathrm{cpd})$, was randomly assigned to each participant and applied consistently throughout the experiment. Given a two-alternative forced-choice (2AFC) orientation discrimination task, participants were asked to press either the left or the right mouse button as soon as they noticed the orientation of the Gabor patch, clockwise or counterclockwise, respectively. In order to ensure a continuous engagement of attention after each behavioral response, participants were also asked to press the mouse button again when the perceived the luminance contrast of Gabor patches starting to decrease. Prior to the actual experiment, participants were given 4 to 8 practice trials of the target task until they became familiar with a mouse-button press corresponding to each stimulus orientation. Response time was measured relative to the onset of the Gabor patch stream. 


\section{EEG recordings and Data analysis}

The Electroencephalogram (EEG) was continuously recorded from 257 electrodes using an Electrical Geodesics (EGI) system. Data were digitized at a rate of $250 \mathrm{~Hz}$, using $\mathrm{Cz}$ as a recording reference. All electrode impedances were kept below $50 \mathrm{kOhms}$ as recommended for the EGI high input impedance amplifier and suggested by empirical observations of signal-to-noise ratios under varying impedance levels (Ferree, Luu, Russell, \& Tucker, 2001). A low-pass filter of $30 \mathrm{~Hz}$ was applied, and epochs of $400 \mathrm{~ms}$ pre- and $7400 \mathrm{~ms}$ postonset of the non-flickering picture cues were extracted. Using the procedure of artifact rejection proposed by Junghöfer et al. (2000), trials with artifacts were identified based on the distribution of statistical parameters of EEG epochs (absolute value, standard deviation, maximum of the differences) extracted across time points and channels. Sensors contaminated with artifacts were replaced by statistically weighted, spherical spline interpolated values. In addition to the artifact control, trials with excessive eye movements, blinks, and other ocular artifacts were dismissed based on visual inspection of the vertical and horizontal electro-oculogram (EOG) computed from a subset of the net sensors. For interpolation and all subsequent analyses, data were arithmetically transformed to the average reference. No significant differences in the number of retained trials or channels were observed for the three emotional contents.

\section{Steady-state VEP analyses}

Artifact free epochs of the voltage data were averaged for the 3 emotional contents preceding the flickering stream of Gabor patches. At each of the resulting 256 source locations, the time-varying amplitude at the stimulation frequency of $14 \mathrm{~Hz}$ was extracted by means of complex modulation (Regan, 1989). This procedure used sine and cosine functions at the stimulation frequency, multiplied with the empirical signal. The products are then low-pass filtered ( $3^{\text {rd }}$ order 1-Hz low-pass Butterworth filter, $12 \mathrm{~dB} / \mathrm{Octave}$ ) and their absolute value is obtained as the modulus of the sine and cosine products (see Müller, Keil, Kissler, \& Gruber, 2001). This results in time-varying amplitude (in microVolts) of the signal at the stimulation frequency. The mean of a 1000-msec segment prior to and post to picture onset was subtracted as baseline. This segment was selected to eliminate any offset related to the IAPS picture onset and offset and result in a fait comparison of the Gaborpatch related activity across conditions.

\section{Statistical Analysis}

Mean ssVEP amplitudes were averaged across a medial-occipital cluster comprising site $\mathrm{Oz}$ and its eight neighbor electrodes [Electrical Geodesics sensors: 118, 119, 120, 126, 127, $128,138,139,140]$, resulting in one ssVEP amplitude value for each emotional content of preceding pictures. The averaged amplitudes were then z-transformed within-subjects to address inter-individual differences. Levels of luminance contrast of gratings within a target Gabor patch stream were determined by the three time segments (1600-2796 ms, 2800$4396 \mathrm{~ms}$, and 4400-5200 ms after the picture onset), corresponding to low-, medium-, and high-contrast. Since there was no difference in ssVEP amplitude in response to low-contrast gratings from the baseline, analyses focused on its comparisons between medium- and highcontrast gratings. Posterior ssVEP differences between the experimental conditions were 
evaluated by means of repeated-measures ANOVAs containing two within-subject factors of emotional content (pleasant, neutral, unpleasant) of pictures and luminance contrast (medium, high) of gratings, and a between-subject factor of spatial frequency (low, high). Interaction effects were followed up by means of (1) t-tests comparing two spatial frequencies for each contrast level of Gabor patches, and (2) contrast analysis for different emotional content of pictures. These post hoc comparisons were conducted to specifically evaluate differences in the overall temporal dynamics of cortical engagement: Evidence of ssVEP differences during high contrast perception supports response gain modulation, differences during medium contrast perception support contrast gain modulation, and main effects of picture content on the ssVEP amplitude across contrasts supports involvement of both gain mechanisms. These analyses allow us to quantify the effects of emotional content of the preceding stimuli and the spatial frequency of the target stimuli. Median behavioral accuracy and reaction times (RTs) of each individual were averaged for emotional content of preceding pictures. Effects of experimental conditions on task performance were examined by means of repeated-measures ANOVA having a within-subject factor of emotional content and a between-subject factor of spatial frequency. The distribution of percentage error on the forced-choice orientation discrimination task was highly non-normal. Therefore, ANOVAs were calculated on rank-transformed error data, but non-transformed values are reported in figures and means. A significance level of .05 (two-tailed) was applied for the main and post-hoc analyses of ssVEP amplitude and behavioral data.

\section{Results}

\section{Behavioral Data}

All participants were able to perform the orientation discrimination task with high accuracy. The average error rate for both spatial frequency and picture cue conditions was $10 \%$. The repeated measures ANOVA revealed the interaction effect of emotional content by spatial frequency on the rank-transformed error data $(\mathrm{F}(2,46)=3.26, \mathrm{p}=0.043)$. A post-hoc contrast analysis indicated that participants tended to more accurately discriminate the orientation of LSF gratings following emotionally arousing than neutral pictures, but the reversed pattern was observed for HSF gratings $(\mathrm{F}(1,47)=4.65, \mathrm{p}=0.036)$. In addition, RTs were measured relative to the Gabor patch stream onset. Across conditions with different spatial frequencies, RTs were significantly higher when target gratings were preceded by unpleasant pictures [mean $R T=1596.10, S D=398.67$ ], compared to pleasant [mean $R T$ $=1555.14, S D=420.26$ ] and neutral stimuli [mean $R T=1558.60$, $S D=380.86$ ] $(\mathrm{F}(1,47)=4.06, \mathrm{p}<0.05)$. However, there was no main effect of spatial frequency or interaction effect of emotional content and spatial frequency. Spearman's rho showed a statistically significant relationship between error rates and RTs for pleasant $(\mathrm{rs}=.56, \mathrm{p}<$ $0.001)$, neutral ( $r s=.66, \mathrm{p}<0.001)$, and unpleasant ( $\mathrm{rs}=.49, \mathrm{p}<0.001)$ conditions, suggesting that no speed/accuracy tradeoff across conditions was observed.

\section{EEG Data}

The target Gabor patch stream reliably evoked steady-state responses, as can be seen from the complex-demodulated ssVEP envelope, which follows the contour of the contrast function (see Figure 3). We observed a main effect of luminance contrast of target Gabor 
patches $(F(1,47)=139.94, \mathrm{p}<0.001)$, showing greater ssVEP amplitudes for the time segment with high contrast compared to medium contrast. As demonstrated by an interaction of contrast with spatial frequency, the ssVEP in response to the target stimulus stream of contrast-varying Gabor patches were differentially modulated by spatial frequency $(F(1,47)$ $=4.84, \mathrm{p}=0.033)$. Post hoc comparisons using independent samples t-tests of the absolute value of the difference between for the high and medium contrast segments revealed that the enhancement for high contrast was most pronounced in the low-spatial frequency condition $(t(47)=2.86, p=0.006)$. No main effect of emotion was observed $(F(2,46)<1.3)$. Of note with respect to the hypotheses of this study, the ssVEP amplitudes of Gabor patches were differentially modulated by the emotional content of the pictures and the spatial frequency of the target patch $(F(2,46)=5.54, p=0.007$; see Figure 4). To examine this interaction, post hoc comparisons were conducted on amplitudes for the low- and high-spatial frequency conditions, for each emotion condition. Specifically, greater amplitudes were observed for the low-spatial frequency target gratings following emotionally arousing pictures (pleasant and unpleasant) than neutral ones ( $\mathrm{p}=0.023$ ), whereas the ssVEP amplitudes of high-spatial frequency targets were greater for those preceded by neutral pictures than emotionally arousing ones $(\mathrm{p}=0.021)$. These effects did not interact with luminance contrast (medium versus high) of the Gabor patch, suggesting a contrast-independent ssVEP enhancement for the low spatial frequency target and general reduction for the high spatial frequency, after viewing emotionally arousing pictures.

\section{Discussion}

Emotionally arousing stimuli have been shown to be motivationally relevant cues and to attract attention and processing resources (see Lang \& Bradley, 2010). Divergent consequences of this prioritized processing have been described in the literature, demonstrating facilitation (e.g., Phelps, et al., 2006) or interference (e.g., Most, et al., 2007) effects of emotion on concurrent or subsequent visual processing. More recently, Bocanegra and Zeelenberg (2009b) demonstrated that the influence of emotional engagement may depend on the physical properties of subsequent visual stimuli. The present study employed ssVEPs to address two major questions regarding the benefits and costs of emotional engagement on subsequent visual processing by measuring the continuous electrocortical responses to a target visual stimulus stream. The target stream, consisting of contrastvarying low- or high-spatial frequency Gabor patches, followed a task-irrelevant picture cue with different emotional content. The first aim in the present study was to characterize the direction of emotion effects (facilitation or interference) on the electrocortical processing of subsequent neutral targets. Our ssVEP results indicate that emotionally arousing stimuli selectively enhance the amount of processing resources allocated toward subsequent lowspatial frequency targets, but attenuate resource allocation toward high-spatial frequency targets. This is consistent with findings regarding the emotional modulation of behavioral contrast sensitivity using fearful face cues (Bocanegra \& Zeelenberg, 2009b), where discrimination performance depended on the spatial frequency of the target grating. Further, the use of highly arousing pleasant and unpleasant pictures in the present study supports the motivational relevance of emotional arousal, but not valence, for modulating subsequent visual cortical engagement. Our ssVEP findings were partly consistent with the behavioral 
data, where (i) response times were prolonged specifically for the unpleasant picture condition, and (ii) error rates mirrored the interaction between emotion and spatial frequency shown in the ssVEP data: visual discrimination tended to be more accurate for gratings following emotional pictures than neural ones only when the gratings were LSF, but not HSF. To the extent that the behavioral response trails the onset of the picture by several seconds, these differences may well reflect a variety of factors in addition to perceptual sensitivity, such as sustained effects of the leading picture on response selection and execution (Ihssen, Heim, \& Keil, 2007). For instance, slowing of RTs after viewing unpleasant pictures has been reported previously, even when the temporal lag between the unpleasant picture and the response spanned several seconds (e.g., Ihssen \& Keil, in press).

The second goal of the current research was to examine neural mechanisms that mediate selective benefits or costs of emotional engagement for the processing of target stimuli with different visual properties, levels of luminance contrast and spatial frequency. This issue was expected to provide evidence on the roles of early resource allocation in emotionmodulated subsequent visual processing, characterized by (a) gain models of attention and/or (b) the visual preferential activation of magnocellular vs. parvocellular channels in lower-tier visual areas.

In terms of gain models of attention, the present findings suggest that the emotional content of picture cues does not alter electrocortical responses to specific contrast ranges but affects all levels equally. Differences in the ssVEP amplitude for target Gabor patches between emotional and neutral picture cue conditions were similar for medium- and high-contrast gratings, respectively, and no difference was observed for low contrast segments, where the ssVEP did not differ from baseline. This supports that the ssVEP amplitude of subsequent target stimuli does not simply reflect contrast or response gain, but rather a combination of both mechanisms. Consistent with a mixed model of gain modulation during sustained attention (Huang \& Dobkins, 2005) and transient/exogenous attention (Ling \& Carrasco, 2006), our results may reflect different stages of visual processing altered by emotional arousal: In a hypothetical account, resource allocation toward subsequent targets first undergoes contrast gain modulation and is followed by response gain modulation at a later processing stage (Huang \& Dobkins, 2005). Such an account is particularly likely in our present design, where contrast and time were confounded, and high contrast always followed low contrast in time. To address this issue, we analyzed the time period of linear contrast reduction, i.e. the waning phase of the present experiment. In this phase, no effects of emotional content on the ssVEP were observed, which may also be attributed to the temporal distance between the picture and the waning part of the Gabor stream. This finding appears to contradict a further alternative explanation of the present findings, in which viewing an arousing picture results in an unspecific increase in overall visual neuronal activity (sometimes referred to as activity gain). However, the hallmark of activity gain, an increase in baseline activity following picture onset, would not be visible in the ssVEP, which solely reflects the aspect of the neural activity that is related to the driving stimulus. Future studies using various combinations of the physical properties of visual stimuli along with measures that do not rely on driving neural masses with a continuous external stimulus may further elucidate neuronal gain mechanisms in the context of emotional engagement. 
We also observed greater electrocortical activity during the processing of low-spatial frequency gratings following emotional, compared to neutral, pictures, whereas a reversed pattern was observed for the targets with high-spatial frequency. These results may be taken to suggest that the processing of emotional stimuli predominantly involves the magnocellular pathway, which is capable of rapidly conveying low-spatial frequency information to emotion-modulated structures such as the amygdala (Amaral, et al., 2003). In this respect, the present study extends previous work in two ways: first, our findings replicate the interaction of emotion and spatial frequency with respect to subsequent perceptual sensitivity. Previous hemodynamic and electrophysiological studies have shown that differential spatial frequencies of face stimuli alter the early perception of emotional expressions. For example, several fMRI studies reported that emotional facial expressions with low-spatial frequency, compared to high-spatial frequency, elicited a greater activation of the amygdala as well as the visual cortex, potentially mediated by the activity of the amygdala (Winston, Vuilleumier, \& Dolan, 2003). In ERP studies, brain wave differences were associated with spatial frequency approximately at $130 \mathrm{~ms}$ in response to emotional faces (P1 component; Pourtois et al., 2005) and at $135 \mathrm{~ms}$ in response to non-facial emotional pictures (sometimes called the N135 component; Carretie, Hinojosa, LopezMartin, \& Tapia, 2007). Second, the results are in line with notions that the two major visual pathways, magnocellular and parvocellular, vary in their sensitivity to emotional arousal. In addition, our behavioral accuracy and ssVEP data may suggest that engaging predominantly one pathway may inhibit the other, respectively. Such an inter-channel inhibition mechanism has also been suggested by Bocanegra and Zeelenberg (2009; 2011): Emotional engagement may selectively enhance the sensitivity of magnocellular channels at the expense of parvocellular channels.

How is a mixed gain modulation of visual perception, subsequent to emotional engagement, associated with magnocellular and parvocellular pathways in the context of stimulus physical properties and arousal state? First, several studies have indicated that contrast gain control is stronger in magnocellular cells than parvocellular cells (e.g., Benardete, Kaplan, \& Knight, 1992; B. Lee, Pokorny, Smith, \& Kremers, 1994). Second, response gain has been demonstrated as a consequence of cholinergic ( $\mathrm{ACH}$ ) modulation (e.g., arousal) of visual information processing in V1 of the macaque, mainly within layer 4C (Soma, Shimegi, Osaki, \& Sato, 2012), where the anatomical convergence of parvocellular and magnocellular inputs occurs (e.g., Lund, Wu, Hadingham, \& Levitt, 1995; Vidyasagar, Kulikowski, Lipnicki, \& Dreher, 2002). Given that the amygdala receives both types of visual input but predominantly projects to the visual cortices via magnocellular connections (Amaral, et al., 2003), the present results for contrast and response gain modulation may allow us to formulate hypotheses regarding neural mechanisms by which emotional engagement influences the activity of distinct visual pathways. For instance, future research may use hemodynamic imaging in conjunction with electrophysiology to examine the extent to which the modulation of time-dependent gain mechanisms by emotion is related to engagement of specific anatomical regions such as the amygdala.

As an unexpected effect, the present data showed an interaction between contrast and spatial frequency irrespective of preceding emotional content. We found greater amplitude differences between medium- and high-contrast gratings for low compared to high spatial 
frequency. Future studies may want to clarify the sensitivity of ssVEPs to a concurrent modulation of contrast and spatial frequency. Caution is warranted when interpreting our behavioral and ssVEP data as purely reflecting magnocellular and parvocellular input, as these systems respond differently to changing combinations of spatial frequency and stimulus contrast. In particular, the parvocellular pathway is thought to convey high-contrast information (Kveraga, Boshyan, \& Bar, 2007) across multiple spatial frequencies (Carretie, et al., 2007), rather than only high spatial frequency information. Moreover, magnocellular and parvocellular pathways differ in their temporal sensitivity, with magnocellular processes being tuned to faster- and parvocellular pathways to slower-paced events, respectively (e.g., Rice \& Gilbert, 1990). Thus, the current design in which visual processing was modulated at a rapid stimulation frequency of $14 \mathrm{~Hz}$ may favor to some extent the magnocellular pathway. This is important because processing speed and stimulus contrast have been shown to interactively affect both pathways. For instance, parvocellular-biased (e.g., HSF) stimuli requiring a high contrast threshold were more easily detected at higher temporal frequencies when a lesion was limited to the magnocellular layers in the LGN (Merigan \& Maunsell, 1993). Despite these limitations, the ssVEP amplitudes in response to HSF stimuli were attenuated only when the gratings were preceded by emotional, but not by neutral cues, suggesting that the HSF response was robust overall. In addition, no main effect of spatial frequency was observed, suggesting that the overall level of the ssVEP was comparable across spatial frequencies.

Taken together, the present study proposes potential parameters that differentially contribute to the effects of emotional engagement on subsequent visual processing: luminance contrast and spatial frequency. This further provides neurophysiological evidence for the interaction between visual and motive systems in the human brain (e.g., Re-entrant modulation of the visual cortex; Keil et al., 2009), possibly pointing to the differential involvement of magnocellular and parvocellular visual pathways in the processing of emotional content and upcoming task-relevant information. Future work comparing different perceptual tasks (e.g., target detection and visual search) may examine the degree to which time- and contrastvarying changes of ssVEP amplitudes reflect emotional modulation of visual processing and/or other task-specific properties.

\section{References}

Amaral DG, Behniea H, Kelly JL. Topographic organization of projections from the amygdala to the visual cortex in the macaque monkey. Neuroscience. 2003; 118(4):1099-1120. [PubMed: 12732254]

Anderson AK, Phelps EA. Lesions of the human amygdala impair enhanced perception of emotionally salient events. Nature. 2001; 411(6835):305-309. [PubMed: 11357132]

Benardete E, Kaplan E, Knight BW. Contrast gain control in the primate retina: P cells are not X-like, some M cells are. Vis Neurosci. 1992; 8(5):483-486. [PubMed: 1586649]

Bocanegra BR, Zeelenberg R. Dissociating emotion-induced blindness and hypervision. Emotion. 2009a; 9(6):865-873. [PubMed: 20001129]

Bocanegra BR, Zeelenberg R. Emotion improves and impairs early vision. Psychol Sci. 2009b; 20(6): 707-713. [PubMed: 19422624]

Bocanegra BR, Zeelenberg R. Emotional cues enhance the attentional effects on spatial and temporal resolution. Psychon Bull Rev. 2011; 18(6):1071-1076. [PubMed: 21901512] 
Bradley B, Mogg K, White J, Groom C, de Bono J. Attentional bias for emotional faces in generalized anxiety disorder. Br J Clin Psychol. 1999; 38(Pt 3):267-278. [PubMed: 10532148]

Bradley M, Lang PJ. Measuring emotion: the Self-Assessment Manikin and the Semantic Differential. J Behav Ther Exp Psychiatry. 1994; 25(1):49-59. [PubMed: 7962581]

Bradley M, Sabatinelli D, Lang PJ, Fitzsimmons JR, King W, Desai P. Activation of the visual cortex in motivated attention. Behav Neurosci. 2003; 117(2):369-380. [PubMed: 12708533]

Brainard DH. The Psychophysics Toolbox. Spat Vis. 1997; 10(4):433-436. [PubMed: 9176952]

Buracas GT, Boynton GM. The effect of spatial attention on contrast response functions in human visual cortex. J Neurosci. 2007; 27(1):93-97. [PubMed: 17202476]

Carrasco M, Ling S, Read S. Attention alters appearance. Nat Neurosci. 2004; 7(3):308-313. [PubMed: 14966522]

Carretie L, Hinojosa JA, Lopez-Martin S, Tapia M. An electrophysiological study on the interaction between emotional content and spatial frequency of visual stimuli. Neuropsychologia. 2007; 45(6): 1187-1195. [PubMed: 17118408]

Chun MM, Potter MC. A two-stage model for multiple target detection in rapid serial visual presentation. J Exp Psychol Hum Percept Perform. 1995; 21(1):109-127. [PubMed: 7707027]

Corbetta M, Miezin FM, Dobmeyer S, Shulman GL, Petersen SE. Attentional modulation of neural processing of shape, color, and velocity in humans. Science. 1990; 248(4962):1556-1559. [PubMed: 2360050]

Desimone, R.; Ungerleider, LG. Neural mechanisms of visual processing in monkeys. In: Boller, F.; Grafman, J., editors. Handbook of Neurophysiology. Vol. 2. Armsterdam: Elsevier; 1989. p. 267-299.

Dolan RJ. Emotion, cognition, and behavior. Science. 2002; 298(5596):1191-1194. [PubMed: 12424363]

Ferrari V, Codispoti M, Cardinale R, Bradley MM. Directed and motivated attention during processing of natural scenes. J Cogn Neurosci. 2008; 20(10):1753-1761. [PubMed: 18370595]

Ferree TC, Luu P, Russell GS, Tucker DM. Scalp electrode impedance, infection risk, and EEG data quality. Clin Neurophysiol. 2001; 112(3):536-544. [PubMed: 11222977]

Huang L, Dobkins KR. Attentional effects on contrast discrimination in humans: evidence for both contrast gain and response gain. Vision Res. 2005; 45(9):1201-1212. [PubMed: 15707928]

Ihssen N, Heim S, Keil A. The costs of emotional attention: affective processing inhibits subsequent lexico-semantic analysis. J Cogn Neurosci. 2007; 19(12):1932-1949. [PubMed: 17892390]

Junghöfer M, Elbert T, Tucker DM, Rockstroh B. Statistical control of artifacts in dense array EEG/MEG studies. Psychophysiology. 2000; 37(4):523-532. [PubMed: 10934911]

Kastner S, Pinsk MA, De Weerd P, Desimone R, Ungerleider LG. Increased activity in human visual cortex during directed attention in the absence of visual stimulation. Neuron. 1999; 22(4):751761. [PubMed: 10230795]

Keil A, Bradley MM, Junghofer M, Russmann T, Lowenthal W, Lang PJ. Cross-modal attention capture by affective stimuli: evidence from event-related potentials. Cogn Affect Behav Neurosci. 2007; 7(1):18-24. [PubMed: 17598731]

Keil A, Ihssen N. Identification facilitation for emotionally arousing verbs during the attentional blink. Emotion. 2004; 4(1):23-35. [PubMed: 15053724]

Keil A, Moratti S, Sabatinelli D, Bradley MM, Lang PJ. Additive effects of emotional content and spatial selective attention on electrocortical facilitation. Cereb Cortex. 2005; 15(8):1187-1197. [PubMed: 15590910]

Keil A, Sabatinelli D, Ding M, Lang PJ, Ihssen N, Heim S. Re-entrant projections modulate visual cortex in affective perception: evidence from Granger causality analysis. Hum Brain Mapp. 2009; 30(2):532-540. [PubMed: 18095279]

Keil A, Smith JC, Wangelin BC, Sabatinelli D, Bradley MM, Lang PJ. Electrocortical and electrodermal responses covary as a function of emotional arousal: a single-trial analysis. Psychophysiology. 2008; 45(4):516-523. [PubMed: 18513365] 
Kim YJ, Grabowecky M, Paller KA, Muthu K, Suzuki S. Attention induces synchronization-based response gain in steady-state visual evoked potentials. Nat Neurosci. 2007; 10(1):117-125. [PubMed: 17173045]

Kveraga K, Boshyan J, Bar M. Magnocellular projections as the trigger of top-down facilitation in recognition. J Neurosci. 2007; 27(48):13232-13240. [PubMed: 18045917]

Lang PJ, Bradley MM. Emotion and the motivational brain. Biol Psychol. 2010; 84(3):437-450. [PubMed: 19879918]

Lang, PJ.; Bradley, MM.; Cuthbert, BN. Technical Report A-6. University of Florida; Gainesville, FL: 2005. International affective picture system (IAPS): Affective ratings of pictures and instruction manual.

Lee B, Pokorny J, Smith VC, Kremers J. Responses to pulses and sinusoids in macaque ganglion cells. Vision Res. 1994; 34(23):3081-3096. [PubMed: 7975341]

Lee D, Koch C, Braun J. Spatial vision thresholds in the near absence of attention. Vision Res. 1997; 37(17):2409-2418. [PubMed: 9381676]

Li X, Lu ZL, Tjan BS, Dosher BA, Chu W. Blood oxygenation level-dependent contrast response functions identify mechanisms of covert attention in early visual areas. Proc Natl Acad Sci U S A. 2008; 105(16):6202-6207. [PubMed: 18413602]

Ling S, Carrasco M. Sustained and transient covert attention enhance the signal via different contrast response functions. Vision Res. 2006; 46(8-9):1210-1220. [PubMed: 16005931]

Lund JS, Wu Q, Hadingham PT, Levitt JB. Cells and circuits contributing to functional properties in area V1 of macaque monkey cerebral cortex: bases for neuroanatomically realistic models. $\mathrm{J}$ Anat. 1995; 187(Pt 3):563-581. [PubMed: 8586556]

Martinez-Trujillo J, Treue S. Attentional modulation strength in cortical area MT depends on stimulus contrast. Neuron. 2002; 35(2):365-370. [PubMed: 12160753]

Morrone MC, Denti V, Spinelli D. Different attentional resources modulate the gain mechanisms for color and luminance contrast. Vision Res. 2004; 44(12):1389-1401. [PubMed: 15066398]

Most SB, Smith SD, Cooter AB, Levy BN, Zald DH. The naked truth: Positive, arousing distractors impair rapid target detection. Cogn \& Emot. 2007; 21:964-981.

Muller MM, Andersen SK, Keil A. Time course of competition for visual processing resources between emotional pictures and foreground task. Cereb Cortex. 2008; 18(8):1892-1899. [PubMed: 18063562]

Muller MM, Picton TW, Valdes-Sosa P, Riera J, Teder-Salejarvi WA, Hillyard SA. Effects of spatial selective attention on the steady-state visual evoked potential in the $20-28 \mathrm{~Hz}$ range. Brain Res Cogn Brain Res. 1998; 6(4):249-261. [PubMed: 9593922]

Ohman A, Flykt A, Esteves F. Emotion drives attention: detecting the snake in the grass. J Exp Psychol Gen. 2001; 130(3):466-478. [PubMed: 11561921]

Pelli DG. The VideoToolbox software for visual psychophysics: transforming numbers into movies. [Journal Article]. Spat Vis. 1997; 10(4):437-442. [PubMed: 9176953]

Pessoa L, McKenna M, Gutierrez E, Ungerleider LG. Neural processing of emotional faces requires attention. Proc Natl Acad Sci U S A. 2002; 99(17):11458-11463. [PubMed: 12177449]

Phelps EA, LeDoux JE. Contributions of the amygdala to emotion processing: from animal models to human behavior. Neuron. 2005; 48(2):175-187. [PubMed: 16242399]

Phelps EA, Ling S, Carrasco M. Emotion facilitates perception and potentiates the perceptual benefits of attention. Psychol Sci. 2006; 17(4):292-299. [PubMed: 16623685]

Pourtois G, Dan ES, Grandjean D, Sander D, Vuilleumier P. Enhanced extrastriate visual response to bandpass spatial frequency filtered fearful faces: time course and topographic evoked-potentials mapping. Hum Brain Mapp. 2005; 26(1):65-79. [PubMed: 15954123]

Raymond JE, Shapiro KL, Arnell KM. Temporary suppression of visual processing in an RSVP task: an attentional blink? J Exp Psychol Hum Percept Perform. 1992; 18(3):849-860. [PubMed: 1500880]

Regan, D. Human Brain Electrophysiology: Evoked Potentials and Evoked Magnetic Fields in Science and Medicine. New York: Elsevier; 1989. 
Reynolds JH, Pasternak T, Desimone R. Attention increases sensitivity of V4 neurons. Neuron. 2000; 26(3):703-714. [PubMed: 10896165]

Sabatinelli D, Flaisch T, Bradley MM, Fitzsimmons JR, Lang PJ. Affective picture perception: gender differences in visual cortex? Neuroreport. 2004; 15(7):1109-1112. [PubMed: 15129155]

Smith SD, Most SB, Newsome LA, Zald DH. An emotion-induced attentional blink elicited by aversively conditioned stimuli. Emotion. 2006; 6:523-527. [PubMed: 16938093]

Soma S, Shimegi S, Osaki H, Sato H. Cholinergic modulation of response gain in the primary visual cortex of the macaque. J Neurophysiol. 2012; 107(1):283-291. [PubMed: 21994270]

Stormark KM, Nordby H, Hugdahl K. Attentional shifts to emotionally charged cues: Behavioural and ERP data. Cognition \& Emotion. 1995; 9:507-523.

Vidyasagar TR, Kulikowski JJ, Lipnicki DM, Dreher B. Convergence of parvocellular and magnocellular information channels in the primary visual cortex of the macaque. Eur J Neurosci. 2002; 16(5):945-956. [PubMed: 12372031]

Vuilleumier P. How brains beware: neural mechanisms of emotional attention. Trends Cogn Sci. 2005; 9(12):585-594. [PubMed: 16289871]

Winston JS, Vuilleumier P, Dolan RJ. Effects of low-spatial frequency components of fearful faces on fusiform cortex activity. Curr Biol. 2003; 13(20):1824-1829. [PubMed: 14561410]

Zeelenberg R, Wagenmakers EJ, Rotteveel M. The impact of emotion on perception: bias or enhanced processing? Psychol Sci. 2006; 17(4):287-291. [PubMed: 16623684] 
A. Contrast gain

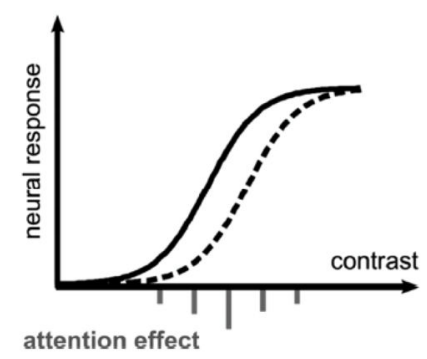

B. Response gain

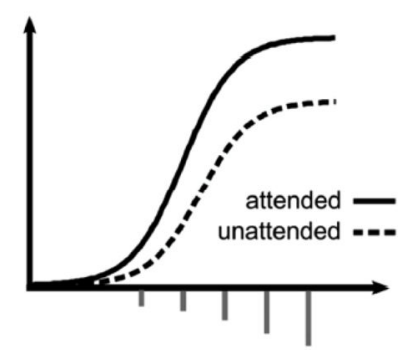

C. Mixed model

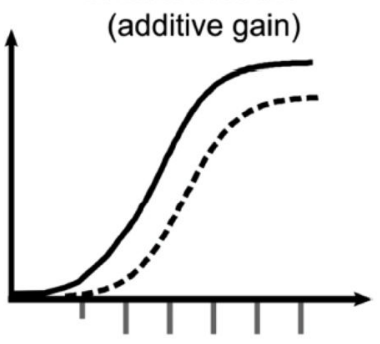

Figure 1.

The contrast, response and mixed (additive) gain models of visual attention. The relative degrees of the shift in the contrast-response function (CRF) mediated by attentional effects at different stimulus contrasts are depicted as the length of vertical gray lines. 


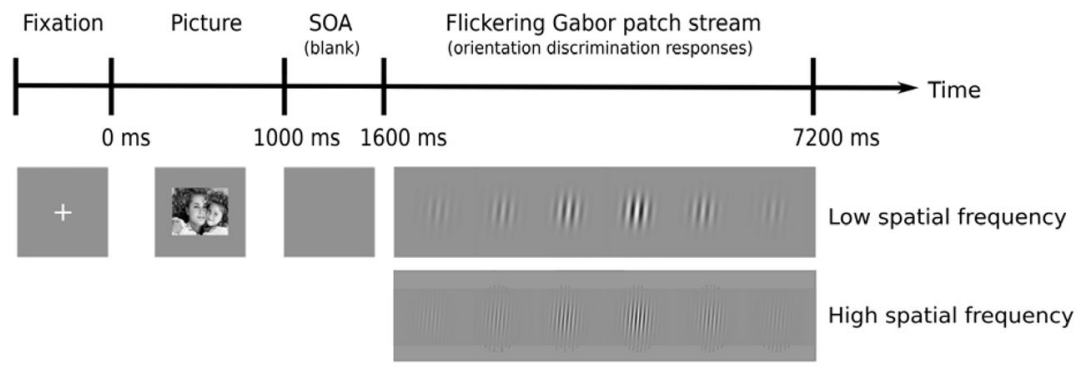

Figure 2.

Schematic illustration of one trial. Inter-trial intervals (fixation) varied randomly between 2500 3000 ms. In each trial, subjects were asked to indicate whether the Gabor patch was tilted to the right or to the left. 

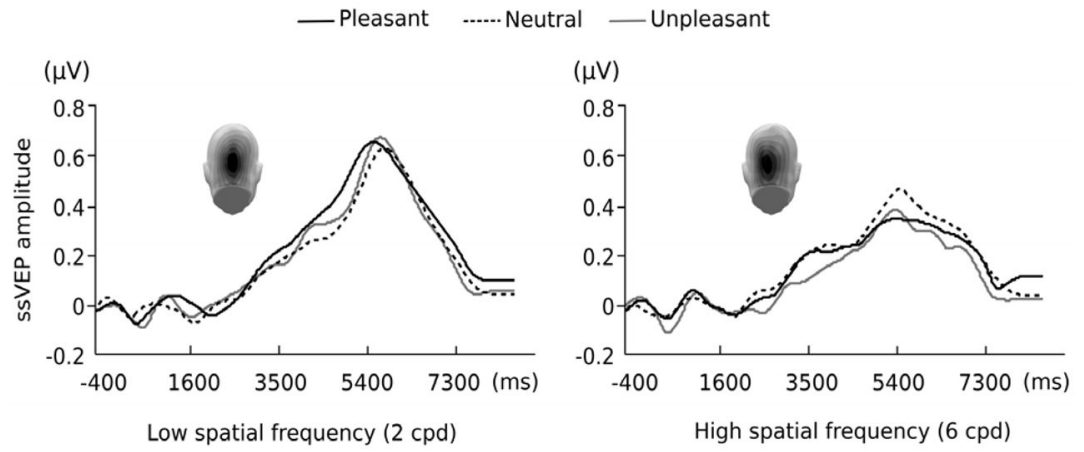

Figure 3.

Time course of 14-Hz ssVEP amplitude in frequency domain obtained by complex demodulation averaged across all subjects for a subset of posterior electrode locations and Oz. Source of the time-averaged ssVEP in the visual cortex is depicted on the topographies. Its contour follows the stimulus contrast of the Gabor patch stream consisting of low and high spatial frequency. Black solid line is for pleasant, black dotted line is for neutral, and gray solid line is for unpleasant pictures preceding the Gabor patch stream. 


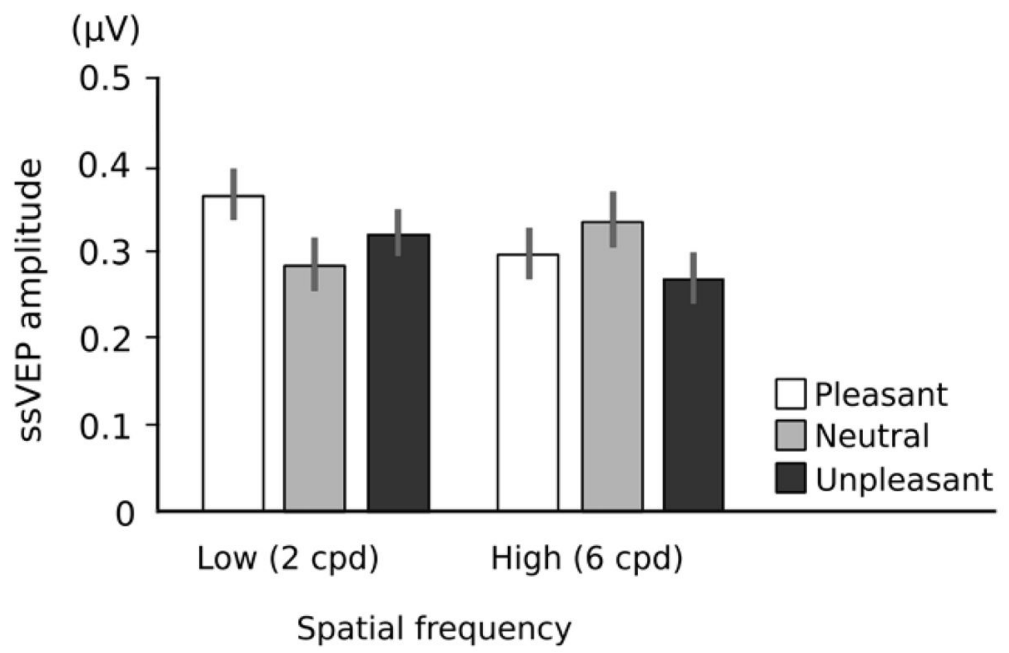

Figure 4.

Interaction plot illustrating the effect of preceding emotional content and spatial frequency on the ssVEP amplitude evoked by the Gabor patch stream. The amplitude was averaged across the medium and high contrast phase (2800 5200 ms after picture onset) in each spatial frequency condition. Values were z-transformed to minimize interindividual variation. 